\title{
The posttraumatic cognitions inventory-Chinese revised: Validation and refinement with a traumatized college sample in Taiwan
}

\author{
Yi-Jen Su, Sue-Huei Chen * \\ Department of Psychology, National Taiwan University, No.1, Sec. 4, Roosevelt Road, Taipei 106, Taiwan \\ Received 28 May 2007; received in revised form 13 November 2007; accepted 13 November 2007
}

\begin{abstract}
The posttraumatic cognitions inventory (PTCI) is a 33-item self-report measure assessing dysfunctional beliefs following trauma. This study reported the factor structure and psychometric properties of the Chinese version of the PTCI (PTCI-C), as well as its relationship with PTSD symptoms. Study participants consisted of 240 traumatized college students in Taiwan. Confirmatory factor analyses indicated adequate replication of the original three-factor structure of the PTCI after eliminating four cross-loaded items. The 29-item PTCI-C was thus developed and its psychometric data were evaluated. This measure displays good internal consistency, test-retest stability, concurrent validity, and discriminative validity. The study findings are discussed in terms of intercultural differences in attribution style and the possible contribution of negative cognitions to PTSD development.
\end{abstract}

(C) 2007 Elsevier Ltd. All rights reserved.

Keywords: Posttraumatic cognition; PTCI; PTSD; Psychometric evaluation; Taiwanese

During the past decade, several cognitive theories have been proposed for explaining the development and persistence of posttraumatic stress disorder (PTSD) (e.g., Brewin, Dalgleish, \& Joseph, 1996; Ehlers \& Clark, 2000; Foa \& Rothbaum, 1998), with the role of trauma induced dysfunctional cognitions being especially emphasized. For instance, two basic dysfunctional cognitions, namely, the world is completely dangerous and I am totally incompetent, were assumed to mediate the development and maintenance of PTSD (Foa \& Rothbaum, 1998). Furthermore, it was proposed that negative appraisals of the trauma and/or its sequelae may produce serious current threat, which is critical to persistent PTSD (Ehlers \& Clark, 2000).

\footnotetext{
* Corresponding author. Tel.: +886 23366 3100; fax: +88622369 9129.

E-mail address: shchen@ntu.edu.tw (S.-H. Chen).
}

Numerous studies have investigated the role of dysfunctional cognitions in PTSD and supported their importance. Negative beliefs as to self and the world in general have displayed a general increase in PTSD patients compared to non-PTSD victims (e.g., Beck et al., 2004; Foa, Ehlers, Clark, Tolin, \& Orsillo, 1999; Startup, Makgekgenene, \& Webster, 2007) and was found to predict subsequent PTSD severity (Dunmore, Clark, \& Ehlers, 2001). Reduction of such beliefs was also found to relate to the reductions in PTSD severity following prolonged exposure therapy (Foa \& Rauch, 2004). In addition, catastrophic appraisal of the trauma and its sequelae reveals an association with PTSD severity (Laposa \& Alden, 2003). Individuals diagnosed with acute stress disorder exhibit more maladaptive appraisals about their functioning following trauma (Nixon \& Byrant, 2005). Furthermore, pretrauma catastrophic thinking could predict posttraumatic stress on follow-up in trainee firefighters (Bryant \& Guthrie, 
2005). Overall, these studies suggest a possible causal relation between negative cognitions and PTSD.

The role of dysfunctional cognitions ensures the importance of adequate and valid assessment of traumarelated beliefs in research of PTSD. The 33-item posttraumatic cognitions inventory (PTCI) was thus developed to assess the negative cognitions induced by traumatic experiences (Foa et al., 1999). The PTCI includes three factors: that is, negative cognitions about self (21 items), negative cognitions about the world (7 items), and self-blame (5 items). The three-factor structure exhibited good replication in different samples, with satisfactory internal consistency for each factor and moderate to high correlations with PTSD severity (Foa et al., 1999). The PTCI has also displayed good discrimination between traumatized individuals with and without PTSD (Beck et al., 2004; Foa et al., 1999; van Emmerik, Schoorl, Emmelkamp, \& Kamphuis, 2006). On the whole, the PTCI exhibits sound psychometric properties.

Notably, Beck et al. (2004) re-examined the psychometric properties of the PTCI in a motor vehicle accident (MVA) sample and obtained somewhat different results. The three-factor structure gained further support after four items were eliminated in order to achieve superior data fit. Moreover, the selfblame subscale displayed worse concurrent and discriminative validity compared with findings of Foa et al. (1999). The different sample characteristics were assumed to account for the inconsistency. More specifically, MVA survivors exhibited less self-blame, while previous study by Foa et al. found that assault victims exhibited excessive self-blame. Accordingly, this study speculated that disparities in negative cognitions may be associated with various types of trauma. Recently, the Dutch version of the PTCI has been developed and evaluated for its psychometric properties (van Emmerik et al., 2006). Although, in contrast with the findings of Beck et al. (2004) and Foa et al. (1999), a few items were found to load on different factors, the results generally supported the three-factor structure of the PTCI.

Owing to lack of relevant tools and of a need for culturally sensitive measures in Taiwan, the purpose of this study was to develop a Chinese version of the PTCI and investigate its psychometric properties. The PTCI is considered to assess self-schema, world-schema, and self-blame thinking. We assume that these constructs, which represent certain forms of fundamental selfknowledge organization, may be shared across cultures. This study thus hypothesized that a similar three-factor structure of the PTCI could be obtained in Taiwanese participants, although some items might be crossloaded as shown in previous studies. Furthermore, this work examined feasibility of the Chinese version of the PTCI for discriminating between individuals with and without PTSD and among individuals suffering various types of trauma. We hypothesized that the Chinese PTCI could differentiate PTSD individuals from noPTSD individuals validly. Furthermore, since the proportion of PTSD individuals is varied by type of trauma (Kessler, Sonnega, Bromet, Hughes, \& Nelson, 1995) and dysfunctional cognitions have been found to raise the risk of developing PTSD as stated above, we hypothesized that the score on the Chinese PTCI would be different among various type of trauma. Specifically, those who had experienced man-made trauma (e.g., sexual assault, physical attack) were assumed to hold stronger dysfunctional beliefs (Foa et al., 1999; Startup et al., 2007) than those who had experienced non-manmade trauma (e.g., natural disaster).

\section{Method}

\subsection{Translation process of the PTCI}

Double-back-translation was performed to avoid language bias. First, the PTCI items were translated into Chinese by the first author. The initial results were then forwarded to two independent translators for backtranslation. Subsequently, both authors carefully reviewed the two initial back-translations and compared them with the original PTCI. Any identified problem areas were reworded in Chinese and sent to another translator for a second back-translation. Finally, result of the back-translation of the affirmed Chinese PTCI was presented to one of the PTCI authors (i.e., E. B. Foa) to confirm its equivalence with the original PTCI.

\subsection{Participants}

Two samples were selected from a large sample and used to examine psychometric properties of the Chinese PTCI. Specifically, factorial validity, internal consistency, concurrent validity, and discriminative validity were investigated in 240 traumatized college students (i.e., the trauma sample). Furthermore, test-retest stability was examined in another sample. The sample characteristics of both samples are presented below.

\subsubsection{Trauma sample}

The factor structure of the Chinese PTCI was examined using 240 college students who had experienced a traumatic incident that satisfied DSM- 
IV PTSD Criterion A (American Psychiatric Association, 1994). These participants were from a large sample of 610 undergraduates at four universities who were enrolled in various psychology courses. Recruitment took place through advertisement posted on the bulletin board in the department of psychology. All participants received course credit points following participation. The large sample comprised $53.9 \%$ females and $44.9 \%$ males, ranging in age from 17 to 37 years old $(M=20.3$, $S D=2.27)$. Three hundred and seventy participants $(60.7 \%)$ did not report a traumatic event that satisfied $D S M-I V$ criteria A, leaving 240 participants $(39.3 \%)$ to constitute the trauma sample for examining factorial validity. The trauma sample comprised $57.6 \%$ female and $42.4 \%$ male. Of all, $52.9 \%$ had experienced criteria A trauma at the time more than 5 years previously, and the majority had experienced natural disasters (37.9\%), accidents $(26.3 \%)$, or physical assaults/abuse (17.1\%).

To examine the discriminative validity of the Chinese PTCI, the trauma sample was separated into PTSD group and no-PTSD groups based on the cut-off point of 15 on the posttraumatic stress diagnostic scale (PDS; Foa, Cashmen, Jaycox, \& Perry, 1997). This score was chosen based on prior discriminant function analysis, which found it to be predictive for the most likely PTSD diagnostic status (Foa et al., 1999). The PTSD group comprised 41 individuals $(17.1 \%)$ who displayed at least moderate PTSD symptom severity (i.e., PDS score $\geq 15$ ). The noPTSD group comprised 199 (82.9\%) individuals who displayed low PTSD symptom severity (i.e., PDS score $<15$ ). Table 1 lists their demographic and clinical information.

\subsubsection{Test-retest sample}

Forty-two additional traumatized college students were administered repeat Chinese PTCI 3-4 weeks after the initial administration $(M=26.2$ days, ranging from 19 to 30 days). The mean age was $19.80 \pm 1.34$ years, and $71.4 \%$ were female. This sample was selected from 350 undergraduates (female $63 \%$ and male $37 \%$ ), who were enrolled in a general psychology course in a large, public university (with a mean age of $19.63 \pm 1.41$ years). All participants received extra course credit following participation. Selection criteria of the test-retest sample were: (a) report at least one traumatic event that satisfied DSM-IV criteria A; (b) agreement to participate the study which investigated the temporal stability of the PTCI and its predictive effect on PTSD (not reported here). Accordingly, the test-retest sample of our study was chosen. The sample was younger than the trauma sample stated above $(t=2.18, p<.05)$, but no difference was noted in sex proportion $\left(\chi^{2}(1)=2.85, p=.09\right)$.

\subsection{Measures}

\subsubsection{Posttraumatic cognitions inventory (PTCI)}

The original PTCI (Foa et al., 1999) is a 33-item measure using a seven-point Likert scale. This measure comprises three subscales: negative cognitions about self, negative cognitions about the world, and selfblame. Scores for these subscales are calculated as the mean item response. The three subscales appear to display sound internal consistency $(\alpha=.87, .88$, and .86 , respectively). The test-retest reliabilities following a 3-week interval for the total score and the three subscales are $.74, .75, .89$ and .89 , respectively. Furthermore, the convergent validity is good. The sensitivity and specificity for PTSD diagnosis are also quite good (sensitivity $=.78 ;$ specificity $=.93$ ). The psychometric data of the Chinese PTCI are presented in Section 2.

\subsubsection{Posttraumatic stress diagnostic scale (PDS)}

The PDS (Foa et al., 1997) is a 49-item self-report measure of PTSD, which assesses presence/absence of all six DSM-IV criteria for PTSD. This scale lists 11 categories of traumatic events and asks respondents to indicate which event bothers them the most. The 17item symptom cluster based on this event is then assessed and the frequency of the PTSD symptoms is rated using a four-point Likert scale. The internal consistency $(\alpha=.92)$ and test-retest reliability $(r=.74$ and .83 for PTSD diagnosis and symptom severity, respectively) is also good. The PDS shows good diagnostic agreement with the Structured Clinical Interview for $D S M-I V$, as well as adequate sensitivity and specificity (.89 and .75 , respectively). The Chinese PDS also exhibits excellent psychometric properties $(\alpha=.91$ for internal consistency; $r=.83$ for test-retest reliability with 3-4 weeks interval) (Su \& Chen, 2006).

\subsubsection{Beck depression inventory-II (BDI-II)}

The BDI-II (Beck, Steer, \& Brown, 1996) is a 21item measure using a four-point Likert scale (0-3), which assesses symptoms of depression severity during the previous 2 weeks, and derives a total score by totaling the ratings of all items (0-63). BDI-II has excellent reliability and validity in American samples (Beck et al., 1996). The internal consistency $(\alpha=.94$, spilt-half $=.91)$ and construct validity was also good in a Taiwanese psychiatric samples (Lu, Che, Chang, \& 
Table 1

Demographic and background variables of the PTSD and no-PTSD groups

\begin{tabular}{|c|c|c|c|}
\hline Variables & $\operatorname{PTSD}(n=41)$ & no-PTSD $(n=199)$ & Group comparisons \\
\hline \multicolumn{4}{|l|}{ Age (in years) } \\
\hline$M( \pm S D)$ & $21.15( \pm 3.16)$ & $20.62( \pm 2.52)$ & $t(234)=1.17, p>.05$ \\
\hline \multicolumn{4}{|l|}{$\operatorname{Sex}(\%)$} \\
\hline Female & 75.6 & 53.8 & $\chi^{2}(1, n=240)=6.60, p<.05$ \\
\hline \multicolumn{4}{|l|}{ Type of trauma (\%) } \\
\hline Accident & 19.5 & 27.6 & $\chi^{2}(8, n=240)=32.49, p<.001$ \\
\hline Disaster & 17.1 & 42.2 & \\
\hline Nonsexual assault & 29.3 & 14.5 & \\
\hline Sexual assault & 7.3 & 1.5 & \\
\hline Sexual abuse & 4.9 & 0.5 & \\
\hline Combat or war zone & 0.0 & 0.5 & \\
\hline Torture & 9.8 & 1.0 & \\
\hline Life-threatening illness & 4.9 & 8.0 & \\
\hline Other & 7.3 & 4.0 & \\
\hline \multicolumn{4}{|l|}{ Time since trauma (\%) } \\
\hline Less than 1 month & 2.5 & 3.0 & $\chi^{2}(3, n=240)=11.19, p<.05$ \\
\hline $1-6$ months & 12.5 & 6.6 & \\
\hline 6 months- 3 years & 32.5 & 13.7 & \\
\hline More than 3 years & 52.5 & 76.7 & \\
\hline \multicolumn{4}{|l|}{ PTS diagnostic scale } \\
\hline$M( \pm S D)$ & $22.93( \pm 6.52)$ & $5.31( \pm 5.00)$ & $t(235)=16.32, p<.001$ \\
\hline
\end{tabular}

Note. Within the type of trauma, the value in boldface represents the trauma in which PTSD and no-PTSD group had significantly different proportions.

Shen, 2002). The BDI-II was used to examine the concurrent validity of the PTCI.

\subsubsection{Beck anxiety inventory (BAI)}

The BAI (Beck \& Steer, 1990) is a 21-item self-report measure for assessing the severity of anxiety symptoms. Individual items are rated on a four-point Likert scale $(0$ 3 ), and the total score is obtained by summing ratings of all items, ranging from 0 to 63. This measure has good reliability and validity (Beck \& Steer, 1990). BDI also demonstrates high internal consistency $(\alpha=.95$, spilthalf $=.91)$ and good convergent validity due to its high correlation with Hamilton Anxiety Rating Scale $(r=.74)$ in a Taiwanese psychiatric sample (Che, Lu, Chen, Chang, \& Lee, 2006). The BAI was used to examine the concurrent validity of the PTCI.

\subsection{Procedure}

After giving informed consent, all 610 participants completed a booklet of measures in a group setting. These measures were written in Chinese and included demographic information, PTCI, and PDS. Most participants completed the procedure in 8-12 min. Participants $(n=370)$ who denied experiencing a criteria A trauma completed the PDS and PTCI by referring to their most stressful life experience and were excluded from further data analyses. A subset of the trauma sample also completed the BDI-II and BAI to examine concurrent validity. This protocol was approved by the Institutional Review Board of the Department of Psychology, National Taiwan University.

\section{Results}

\subsection{Confirmatory factor analyses}

First, Kaiser's measure of sampling adequacy was examined to determine data adequacy for factor analysis. The sampling adequacy value was .95 , making it ideal for further analyses. EQS for Windows 6.1 program (Bentler \& Wu, 2003) was used for confirmatory factor analysis (CFA). As expected of a nonclinical sample, PTCI scores were positively skewed, as indicated by high-normalized Mardia's value (i.e., 62.27). This work thus adopted the robust maximum-likelihood estimation procedure because it adjusts for the data that are not normally distributed. Model fit was evaluated via examining Satorra-Bentler scaled Chi-square (S-B $\chi^{2}$ ), comparative fit index (CFI), non-normed fit index (NNFI), incremental fit index (IFI), and root-mean-square error of approxima- 
Table 2

Standardized item factor loadings of the revised 29-item Chinese version of posttraumatic cognitions inventory

\begin{tabular}{|c|c|}
\hline Item and factor & Factor loading \\
\hline \multicolumn{2}{|l|}{ Factor 1: negative cognitions about self } \\
\hline 2. I cannot trust that I will do the right thing & .756 \\
\hline 3. I am a weak person & .663 \\
\hline 4. I will not be able to control my anger and will do something terrible & .655 \\
\hline 5. I cannot deal with even the slightest upset & .733 \\
\hline 6. I used to be a happy person but now I am always miserable & .683 \\
\hline 9. I feel dead inside & .805 \\
\hline 12. I am inadequate & .803 \\
\hline 14. If I think about the event, I will not be able to handle it & .678 \\
\hline 17. I will never be able to feel normal emotions again & .830 \\
\hline 20. I have permanently changed for the worse & .819 \\
\hline 21. I feel like an object, not a person & .760 \\
\hline 25. I have no future & .762 \\
\hline 26. I can't stop bad things from happening to me & .570 \\
\hline 28. My life has been destroyed by the trauma & .807 \\
\hline 30. My reactions since the event show that I am a lousy coper & .842 \\
\hline 33. I feel like I do not know myself anymore & .832 \\
\hline 35. I cannot rely on myself & .798 \\
\hline 36. Nothing good can happen to me anymore & .834 \\
\hline \multicolumn{2}{|l|}{ Factor 2: negative cognitions about the world } \\
\hline 7. People cannot be trusted & .818 \\
\hline 8. I have to be on guard all the time & .805 \\
\hline 10. You can never know who will harm you & .776 \\
\hline 11. I have to be especially careful because you never know what can happen next & .600 \\
\hline 18. The world is a dangerous place & 689 \\
\hline 23. I cannot rely on other people & 687 \\
\hline 27. People are not what they seem & 699 \\
\hline \multicolumn{2}{|l|}{ Factor 3: self-blame } \\
\hline 1. The event happened because of the way I acted & .757 \\
\hline 15. The event happened to me because of the sort of person I am & .877 \\
\hline 19. Somebody else would have stopped the accident from happening & .551 \\
\hline 31. There is something about me that made the event happen & .863 \\
\hline
\end{tabular}

tion (RMSEA). Conventionally, the model fit is acceptable if the CFI, NNFI, and IFI are .90 or greater and the RMSEA is below .08.

In examining the original three factors specified by Foa et al. (1999), this investigation assigned 21, 7, and 5 items to Factor 1 (negative cognitions about self; SELF), Factor 2 (negative cognitions about the world; WORLD), and Factor 3 (self-blame; BLAME), respectively. Each item was constrained to load only on one factor. Consequently, the proposed model had unsatisfactory data fit: S-B $\chi^{2} \quad(492)=1023.83$, $p<.001 ; \mathrm{CFI}=.88 ; \mathrm{NNFI}=.87 ; \mathrm{IFI}=.88 ; \mathrm{RMSEA}$ $=.067$. The Lagrange multiplier test (LMT) was then conducted to improve the model fit. The result demonstrated that items $16,22,24$, and 29 yielded higher modification index and cross-loaded on two factors. Items 16, 24, 29 belonged to the SELF subscale and item 22 belonged to the BLAME subscale. These items were eliminated and CFA was conducted again using the revised model. As a result, fit indices were acceptable, that is, S-B $\chi^{2}(374)=731.38, p<.001$; $\mathrm{CFI}=.91 ; \mathrm{NNFI}=.90 ; \mathrm{IFI}=.91 ;$ and $\mathrm{RMSEA}=.063$. The standardized item loadings for the final model ranged from .55 to .88 (see Table 2). The revised 29item Chinese PTCI (abbreviated as PTCI-C) comprised a 18-item SELF subscale, a 7-item WORLD subscale, and a 4-item BLAME subscale.

\subsection{Internal consistency}

The internal consistency of the PTCI-C was assessed by calculating corrected item-total correlations and Cronbach's alpha coefficients. The corrected item-total correlations for the three PTCI-C subscales and Total Score were as follows: SELF (range $=.56$ to .81 ), WORLD (range $=.59$ to .74 ), BLAME (range $=.50$ to .80 ), and Total Score (range $=.45$ to .78 ). Moreover, Cronbach's alpha coefficients for the three PTCI-C 
Table 3

Spearman correlations of the Chinese PTCI, PDS, BDI-II and BAI

\begin{tabular}{|c|c|c|c|c|}
\hline Measure & PTCI-self & PTCI-world & PTCI-blame & PTCI-total \\
\hline \multicolumn{5}{|l|}{ PTCI } \\
\hline PTCI-self & - & & & \\
\hline PTCI-world & .74 & - & & \\
\hline PTCI-blame & .76 & .64 & - & \\
\hline PTCI-total & .97 & .86 & .84 & \\
\hline \multicolumn{5}{|l|}{ PTSD measure } \\
\hline PDS-intrusion & .61 & .42 & .61 & .61 \\
\hline PDS-avoidance & .57 & .53 & .54 & .60 \\
\hline PDS-arousal & .69 & .65 & .60 & .72 \\
\hline PDS-total & .68 & .59 & .64 & .71 \\
\hline Depression measure, BDI-II & .72 & .53 & .52 & 69 \\
\hline Anxiety measure, BAI & .48 & .29 & .43 & .46 \\
\hline
\end{tabular}

Note. All values listed are significant at $p<.01$ level. PDS: posttraumatic diagnostic scale; BDI-II: beck depression inventory-II; BAI: beck anxiety inventory.

subscales and total scale were .96 (SELF), .89 (WORLD), .83 (BLAME), and .96 (total scale). To summarize, the PTCI-C displays excellent internal consistency.

\subsection{Test-retest stability}

The 1-month test-retest reliabilities of the PTCI-C were as follows: SELF, $r=.80$; WORLD, $r=.80$; BLAME, $r=.75$; and Total Score, $r=.81$ (all $p$ 's $<.001)$. The results showed that the PTCI-C has good test-retest stability.

\subsection{Concurrent validity}

A subset $(n=87)$ of the trauma sample was used to examine the concurrent validity of the PTCI-C. This subset comprised $51.7 \%$ female and $48.3 \%$ male, ranging from 18 to 31 years old $(M=21.12, S D=3.07)$. No significant differences in age, sex, PDS Total Score, and PTCI-C Total Score were identified between these subjects and the rest of the trauma sample.

Table 3 lists correlations of the PTCI-C with PDS, BDI-II and BAI. Parallel to the previous findings (Beck et al., 2004; Foa et al., 1999), the PTCI-C Total Score and all subscales scores had moderate to high correlations with PTSD severity and different PTSD symptom clusters (all $p$ 's $<.001$ ). A similar pattern was observed for depression severity assessed by the BDI-II (all $p$ 's $<.001$ ) and consistent with those found by Foa et al. (1999) and Beck et al. (2004). Moreover, the PTCI-C Total Score and subscale scores showed significant but somewhat low to moderate correlations with anxiety severity assessed by the BAI (all $p$ 's $<.01$ ).
Partial correlations between the PTCI-C and PTSD severity were calculated, while partialling out both the BDI-II and BAI scores. The result indicated that the PTCI-C Total Score was still moderately correlated with the PDS Total Score $(\mathrm{pr}=.46, p<.001)$ even when controlling for depression and anxiety severity. Partial correlation patterns were also observed in the SELF $(\mathrm{pr}=.39)$, WORLD $(\mathrm{pr}=.38)$, and BLAME subscales $(\mathrm{pr}=.45)($ all $p$ 's $<.001)$. Taken together, the above data demonstrated that the PTCI-C has satisfactory concurrent validity.

\subsection{Discriminative validity}

The following analyses were performed to investigate the discriminative validity of the PTCI-C. The $t$ test and analysis of variance (ANOVA) on the PTCI-C Total Score and subscale scores were conducted to examine the differences between or among the groups of interest. Moreover, discriminant function analyses were used to examine how accurately the individuals in the PTSD group could be identified from the total trauma sample.

\subsection{Difference between PTSD and no-PTSD groups}

The differences between the PTSD and no-PTSD groups on the PTCI-C Total Score and subscale scores were examined. It is noted that both groups differed in sex, time passed since the trauma, and type of trauma (see Table 1); however, $t$-test instead of ANCOVA was used here for two reasons: (a) generally speaking, ANCOVA is appropriate when the groups do not differ 
Table 4

Means and SDs for PTCI subscales and total score by PTSD, no-PTSD group, and trauma type

\begin{tabular}{|c|c|c|c|c|c|c|c|c|}
\hline \multirow[t]{2}{*}{ PTCI subscales } & \multicolumn{2}{|c|}{ PTCI-self } & \multicolumn{2}{|c|}{ PTCI-world } & \multicolumn{2}{|c|}{ PTCI-blame } & \multicolumn{2}{|c|}{ PTCI-total } \\
\hline & $M$ & $S D$ & $M$ & $S D$ & $M$ & $S D$ & $M$ & $S D$ \\
\hline PTSD & 3.28 & 0.93 & 4.40 & 0.92 & 3.80 & 1.23 & 104.95 & 24.13 \\
\hline No-PTSD & 1.77 & 0.87 & 2.92 & 1.29 & 2.05 & 1.32 & 60.54 & 26.02 \\
\hline \multicolumn{9}{|l|}{ Univariate results } \\
\hline$t^{\mathrm{a}}$ & \multicolumn{2}{|c|}{9.97} & \multicolumn{2}{|c|}{8.66} & \multicolumn{2}{|c|}{7.83} & \multicolumn{2}{|c|}{10.07} \\
\hline$p$ & \multicolumn{2}{|c|}{.000} & \multicolumn{2}{|c|}{.000} & \multicolumn{2}{|c|}{.000} & \multicolumn{2}{|c|}{.000} \\
\hline Cohen's $d$ & \multicolumn{2}{|c|}{1.72} & \multicolumn{2}{|c|}{1.49} & \multicolumn{2}{|c|}{1.35} & \multicolumn{2}{|c|}{1.73} \\
\hline \multicolumn{9}{|l|}{ Trauma type } \\
\hline 1. Accidents & 1.95 & 0.86 & 3.22 & 1.27 & 2.47 & 1.39 & 67.57 & 26.66 \\
\hline 2. Disasters & 1.79 & 0.93 & 2.87 & 1.28 & 1.48 & 0.87 & 58.21 & 26.91 \\
\hline 3. Nonsexual assaults & 2.52 & 1.30 & 3.76 & 1.43 & 3.48 & 1.50 & 85.57 & 34.89 \\
\hline 4. Sexual assaults & 2.99 & 1.47 & 4.35 & 0.92 & 3.94 & 1.78 & 100.11 & 34.62 \\
\hline 5. Life-threatening illness & 1.86 & 0.78 & 2.68 & 1.19 & 2.25 & 0.97 & 61.22 & 22.89 \\
\hline \multicolumn{9}{|l|}{ Univariate results } \\
\hline$F^{\mathrm{b}}$ & \multicolumn{2}{|c|}{6.33} & \multicolumn{2}{|c|}{6.19} & \multicolumn{2}{|c|}{25.63} & \multicolumn{2}{|c|}{10.14} \\
\hline$p$ & \multicolumn{2}{|c|}{.000} & \multicolumn{2}{|c|}{.000} & \multicolumn{2}{|c|}{.000} & \multicolumn{2}{|c|}{.000} \\
\hline$\eta^{2}$ & \multicolumn{2}{|c|}{.101} & \multicolumn{2}{|c|}{.101} & \multicolumn{2}{|c|}{.305} & \multicolumn{2}{|c|}{.150} \\
\hline Group comparisons & \multicolumn{2}{|c|}{$\begin{array}{l}3^{*}, 4^{*}>1 ; 3^{* *} \\
4^{* *}>2 ; 4^{*}>5\end{array}$} & \multicolumn{2}{|c|}{$\begin{array}{c}3^{* *}, 4^{*}>2 ; 3^{*} \\
4^{*}>5\end{array}$} & \multicolumn{2}{|c|}{$\begin{array}{c}1 * * *, 3 * * * \\
4 * * *>2 ; 3^{* * *} \\
4^{* *}>1 ; 3 * * \\
4^{* *}>5\end{array}$} & $\begin{array}{r}3 *, 4 \\
4 * * *>\end{array}$ & $\begin{array}{l}* * * \\
* *>5\end{array}$ \\
\hline
\end{tabular}

Note. $* p<.05 ; * * p<.01 ; * * * p<.001$.

a d.f. $=238$.

${ }^{\mathrm{b}}$ d.f. $=(4,227)$.

on the covariate; and (b) there lacks good reasons to believe that the group differences on these variables (i.e., sex, time passed since the trauma, and type of trauma) are truly accounted for by chance, but this is a valid condition for performing ANCOVA (Miller \& Chapman, 2001). Sex, time passed since the trauma, and type of trauma are all possible risk factors for PTSD, and thus, the discrepancy between the PTSD and noPTSD groups on these variables would be preexisting and non-random rather than occurring by chance. As indicated in Table 4 , a series of $t$-test found that the PTSD group scored significantly higher than no-PTSD group on the PTCI-C Total Score. Also, the mean SELF, WORLD, and BLAME scores of the PTSD group were significantly higher than those of no-PTSD group.

\subsection{Identification of PTSD cases in traumatized individuals}

Discriminant function analyses found that the three PTCI-C subscales classified $81.7 \%$ of traumatized college students as individuals with or without PTSD, Wilks's $\lambda=.69, \chi^{2}(3)=86.98, p<.001$. Sensitivity was .83 and Specificity was .81 . Results resemble those obtained by Foa et al. (1999) and slightly exceed those of other studies (Beck et al., 2004; van Emmerik et al., 2006).

\subsection{Differences of PTCI-C among various types of trauma}

In examining the discriminative validity of the PTCI$\mathrm{C}$ for types of trauma, participants' traumas were regrouped into six categories due to the low frequencies of certain trauma types (see Table 1): (a) accidents ( $n=67)$; (b) disasters $(n=91)$; (c) nonsexual assault $(n=47)$ including nonsexual assault by a family member or a known person $(n=25)$, nonsexual assault by a stranger $(n=16)$ and torture $(n=6)$; (d) sexual assault $(n=9)$ including sexual assault by a family member or a known person $(n=4)$, sexual assault by a stranger $(n=2)$ and sexual abuse $(n=3)$; (e) lifethreatening illness $(n=18)$; (f) bereavement $(n=8)$ including the death of a family member $(n=5)$ and the suicide of a friend $(n=3)$. A series of one-way ANOVAs was conducted, and the revised trauma type instead of the original one was used for group classification. The bereavement group was excluded for analysis because it may not clearly satisfy criteria A1 and A2 of PTSD. 
As Table 4 shows, differences on the PTCI-C Total Score among the trauma groups were found. Post hoc Tukey tests revealed that the nonsexual and sexual assault groups had significantly greater PTCI-C Total Scores than the remaining groups. One-way ANOVAs also identified significant differences among trauma groups on the SELF, WORLD, and BLAME subscale. Post hoc Tukey tests showed that the mean SELF and WORLD scores of the nonsexual and sexual assault groups significantly exceeded those of the accident and disaster groups. The mean SELF score of the sexual assault group was also higher than that of the lifethreatening illness group. The disaster survivors displayed the lowest mean BLAME score than that of the remaining groups except the life-threatening group. In contrast, the assault victims displayed the highest mean BLAME scores across the groups.

\section{Discussion}

This paper describes the psychometric properties and initial validation of the Chinese revised PTCI. Based on the CFA, four items of the PTCI were eliminated leaving 29 items. Overall fit indices suggested that this new 29-item PTCI-C had acceptable fit. The original three-factor structure of the PTCI proposed by Foa et al. (1999) gained further support in Taiwanese sample, as similar to the factorial structure found in the U.S. (Beck et al., 2004) and Dutch samples (van Emmerik et al., 2006). The internal consistency reliability, 1-month temporal stability, concurrent validity, and discriminative validity were good. The PTCI-C also displayed imperative and meaningful predictions regarding posttraumatic stress symptoms. To our knowledge, this is the first work to investigate factor structure and psychometric properties of the PTCI with a Chinese population and resulted positively.

In the current study, the four removed items were 16, 22, 24, and 29 due to their cross-loadings across different factors. Similarly, Beck et al. (2004) eliminated four cross-loaded items (i.e., items 2, 4, 24, and $29)$ to improve the model fit. Item 24 , "I feel isolated and set apart from others", and Item 29, "There is something wrong with me as a person", were removed in both studies owing to their nonspecificity to the SELF factor. Our data indicate that both items had extra yet small loadings on the WORLD factor. They were thus eliminated rather than reassigned to the WORLD subscale. Furthermore, Foa et al. (1999) suggested that the SELF subscale could be shortened without compromising its psychometric properties. This was demonstrated by our data, in that the abbreviated 18- item SELF subscale still yields sound psychometric properties.

Item 22 of the original BLAME scale (i.e. "Somebody else would not have gotten into this situation") was also eliminated given that it loaded predominantly on the SELF factor rather than the BLAME factor. As Table 2 shows, the constitution of the revised BLAME subscale somehow contradicts the previous findings (i.e., Beck et al., 2004; Foa et al., 1999; van Emmerik et al., 2006), that is, 4 items versus 5 items, respectively. Moreover, the revised 4-item BLAME subscale primarily indicates that the victims assign the cause of trauma to themselves. Inconsistencies in the findings can be understood as resulting from cultural differences in causal attribution. Choi, Nisbett, and Norenzayan (1999) proposed that, as compared with Westerners, East Asians hold stronger "situationism" or beliefs in the importance of the behavioral context. That is, East Asians tend to view the world and reason holistically, and attribute causality to interactions between objects and the world. In other words, they may be highly aware of the various situational and causal factors that influence their judgments and behaviors. Accordingly, Chinese victims, even when holding self-blame attributions, would still consider the possibility of others encountering a similar situation. This may account for the finding that the item 22 did not load on the BLAME factor. From the above discussion, we also infer that Chinese victims may assign less responsibility to themselves in causing trauma than Western victims do. However, this may need future investigation due to that no difference was noted on the mean BLAME subscale score between our finding and those of previous studies (Beck et al., 2004; Startup et al., 2007).

In addition to the aforementioned cultural concerns, it may need to take the functions of blaming into consideration. For instance, Startup et al. (2007) recently proposed that the BLAME subscale was ambiguous in discriminating characterological versus behavioral self-blame, because that it may hold protective and harmful effects on self. They suggest that the BLAME subscale be revised to specifically assess behavioral self-blame. Overall, questions about the original BLAME subscale per se have been raised in different cultures and these doubts need to be further investigated. To date, however, the revised 4-item BLAME subscale would be more suitable for a Chinese population.

This work also examined negative cognitions around different traumas. van der Kolk (1996) suggested that dysfunctional cognitions may vary with trauma type. 
This was supported by our findings that differences among various trauma types occurred in the PTCI-C Total Score as well as three subscales, and were also demonstrated by previous studies (Foa et al., 1999; Startup et al., 2007). Moreover, as Table 4 shows, the BLAME subscale displays the best discrimination ability among three subscales based on effect size index $\left(\eta^{2}\right)$, even more than that of the PTCI-C Total Score.

The present study found that disaster survivors tend to display the lowest means for all PTCI-C subscales. Since most survivors identified the Taiwan Chi-Chi earthquake that measured 7.3 on the Richter scale and occurred over 6 years ago as their most distressing trauma, we assumed that these people have recovered from post-disaster distress after such a long period of time. Furthermore, accident survivors held significantly less self-blame than assault victims, confirming the proposition of Beck et al. (2004). The fact that sexual assault victims scored highest in all the PTCI-C subscales verified our hypothesis that individuals suffered man-made trauma hold stronger dysfunctional belief than those who suffered non-man-made trauma. This is also in line with the findings of Foa et al. (1999) and Startup et al. (2007). So far, it has been evident that the sexual and physical assault victims tend to develop PTSD (Kessler et al., 1995). Therefore, it is interesting to examine associations of assault exposure, posttraumatic cognitions, and incidence of PTSD. Dysfunctional beliefs held by assault victims may interfere with trauma processing and block the integration of trauma memory, further leading to the development and continuance of PTSD. For example, people who frequently think "I'm incapable of dealing with trauma" would tend to avoid activities or places that remind them of the trauma. This coping strategy might prevent trauma victims from contacting with traumarelated situations and generating alternative adaptive thoughts, thus decreasing the chance of processing traumatic memory.

Our study had certain limitations that should be addressed in future studies on the PTCI-C. Current results may have limited generalizability, since they reflect responses of a traumatized college student sample which is likely to be different from clinical populations in extent of PTSD. A larger clinical sample thus is required to conduct CFA on a PTSD patient sample. Furthermore, use of a homogeneous trauma sample may be also considered. Beck et al. (2004) proposed that sample heterogeneity in Foa et al. (1999) study may influence the initial psychometric data for the PTCI, thus using an MVA sample to re-examine the factorial structure. Likewise, cognitive features of PTSD victims are assumed to differ following different types of traumas. For example, self-blame is considered a salient feature of PTSD following sexual assault but was mild and irrelevant to posttraumatic symptomatology in MVA survivors (Beck et al., 2004). Accordingly, further studies are needed to address whether cognitive feature of victims following varied traumas will influence the factorial structure of the PTCI in general and PTCI-C in specific. Overall, more studies on various samples with heterogeneous traumas may be required to enhance the research and clinical utility of the PTCI-C.

\section{Acknowledgments}

The authors are grateful to Dr. Edna B. Foa for allowing us to translate the posttraumatic cognitions inventory and posttraumatic stress diagnostic scale into Chinese for our research in Taiwan. We also thank all participants of this study for their substantial help in data collection. The authors would like to thank the National Science Council of Taiwan for grant support (No. NSC 94-2625-Z-002-018).

\section{References}

American Psychiatric Association. (1994). Diagnostic and statistical manual of mental disorders (4th ed.). Washington, DC: APA Press.

Beck, A. T., \& Steer, R. A. (1990). Manual for the beck anxiety inventory. Taipei: Chinese Behavioral Science Corporation.

Beck, A. T., Steer, R. A., \& Brown, G. K. (1996). Manual for the beck depression inventory-II. Taipei: Chinese Behavioral Science Corporation.

Beck, J. G., Coffey, S. F., Palyo, S. A., Gundmundsdottir, B., Miller, L. M., \& Colder, C. R. (2004). Psychometric properties of the posttraumatic cognitions inventory (PTCI): a replication with motor vehicle accident survivors. Psychological Assessment, 16, 289-298.

Bentler, P. M., \& Wu, E. J. C. (2003). EQS structural equations program (Version 6.1) (Computer software). Encino, CA: Multivariate Software.

Brewin, C. R., Dalgleish, T., \& Joseph, S. (1996). A dual representation theory of posttraumatic stress disorder. Psychological Review, 103, 670-686.

Bryant, R. A., \& Guthrie, R. M. (2005). Maladaptive appraisals as a risk factor for posttraumatic stress disorder. Psychological Science, 16, 749-752.

Che, H. H., Lu, M. L., Chen, H. C., Chang, S. W., \& Lee, Y. J. (2006). Validation of the Chinese version of the beck anxiety inventory. Formosan Journal of Medicine, 10, 447-454.

Choi, I., Nisbett, R. E., \& Norenzayan, A. (1999). Causal attribution across cultures: variation and universality. Psychological Bulletin, $125,47-63$.

Dunmore, E., Clark, D. M., \& Ehlers, A. (2001). A prospective investigation of the role of cognitive factors in persistent post- 
traumatic stress disorder after physical or sexual abuse. Behavior Research and Therapy, 39, 1063-1084.

Ehlers, A., \& Clark, D. M. (2000). A cognitive model of posttraumatic stress disorder. Behavior Research and Therapy, 38, 319-345.

Foa, E. B., \& Rothbaum, B. O. (1998). Treating the trauma of rape: cognitive behavior therapy for PTSD. New York: Guilford Press.

Foa, E. B., Cashmen, L. A., Jaycox, L., \& Perry, K. (1997). The validation of a self-report measure of posttraumatic stress disorder: the posttraumatic diagnostic scale. Psychological Assessment, 9, 445-451.

Foa, E. B., Ehlers, A., Clark, D. M., Tolin, D. F., \& Orsillo, S. M. (1999). The posttraumatic cognitions inventory (PTCI): development and validation. Psychological Assessment, 11, 303-314.

Foa, E. B., \& Rauch, S. A. M. (2004). Cognitive changes during prolonged exposure versus prolonged exposure plus cognitive restructuring in female assault survivors with posttraumatic stress disorder. Journal of Consulting and Clinical Psychology, 72, 79884.

Kessler, D. C., Sonnega, A., Bromet, E., Hughes, M., \& Nelson, C. B. (1995). Posttraumatic stress disorder in the national comorbidity survey. Archives of General Psychiatry, 52, 1048-1060.

Laposa, J. M., \& Alden, L. E. (2003). Posttraumatic disorder in the emergency room: exploration of a cognitive model. Behavior Research and Therapy, 41, 49-65.
Lu, M. L., Che, H. H., Chang, S. W., \& Shen, W. W. (2002). Reliability and validity of the Chinese version of the beck depressive inventory-II. Taiwanese Journal of Psychiatry, 16, 301-310.

Miller, G. A., \& Chapman, J. P. (2001). Misunderstanding analysis of covariance. Journal of Abnormal Psychology, 110, 40-48.

Nixon, R. D., \& Byrant, R. A. (2005). Are negative cognitions associated with severe acute trauma response? Behaviour Change, $22,22-28$.

Startup, M., Makgekgenene, L., \& Webster, R. (2007). The role of self-blame for trauma as assessed by the posttraumatic cognitions inventory (PTCI): a self-protective cognition? Behaviour Research and Therapy, 45, 395-403.

$\mathrm{Su}$, Y. J., \& Chen, S. H. (2006). The prevalence of traumatic events and current PTSD in Taiwanese college students. Paper presented at the 45th annual conference of the Taiwanese Psychological Association.

van der Kolk, B. A. (1996). The complexity of adaptation to trauma: self-regulation, stimulus discrimination, and character logical development. In: van der Kolk, B. A., McFarlane, A. C., \& Weisaeth, L. Eds. Traumatic stress. (pp.182-213). .

van Emmerik, A. A. P., Schoorl, M., Emmelkamp, P. M. G., \& Kamphuis, J. H. (2006). Psychometric evaluation of the Dutch version of the posttraumatic cognitions inventory (PTCI). Behavior Research and Therapy, 44, 1052-1065. 